\title{
Biophysical Modeling of the Relative Relationships between the Brain and the "Hypothetical Secondary Brains"
}

\author{
Janos Vincze* and Gabriella Vincze-Tiszay \\ Health Human International Environment Foundation, Budapest, Hungary \\ *Corresponding author details: Janos Vincze ; ndp@t-online.hu
}

\begin{abstract}
When we place humans at the peak of the phylogeny and evolution of the living world, we primarily rely on the extraordinary development of their nervous system and brain. The development of the human brain is far from complete, and its immense capacity is not yet exhausted. In particular, new, more modern learning methods are needed to make brain performance more effective by learning about and taking greater account of age-specific characteristics. Learning methods can only be used in certain cases. They cannot replace, but merely complement the acquisition of knowledge, practice, problem and task solving based on experience or observation. Quantitative growth alone is mainly characteristic of those parts of the brain that are called called the hypothetical secondary brain. These consist of constant (stable) topograms and functiograms born with us. During a person's life, they barely change, these provide the centers of the reflexes we are born with and the neural activities associated with them. Memorization is closely related to the formation of unstable topograms and their persistence over time. The significant difference occurs in qualitative growth, which is not characteristic of hypothetical secondary brains, while in the central nervous system it plays a decisive role. Qualitative growth creates the elements and conditions of psychic regulation in humans.
\end{abstract}

Keywords: hypothethical secondary brain; topograms; functiogrmas; quantitative growth; qualitative growth

\section{INTRODUCTION}

In almost all multicellular animals - and this is especially true for humans - there is a specific system, the nervous system, at the service of perceiving external stimuli and organizing stimulus responses. The more advanced a nervous system is, the more versatile the relationship between the environment and the living organism.

During the many millions of years of evolution of the nervous system, three basic stages of development prevailed: 1 . increasing centralization of the nervous system; 2 . conglomeration of nerve elements and sensory organs in the head; 3 . the increasing development of the cortical areas of the cerebrum, the growth of the gray matter - the cerebral cortex consisting of neurons. [1]

At the stage of phylogenetic development, stimulusreceiving receptors appear first and then connect to the executing cells, leading to the formation of the diffuse nervous system. The cells conglomerate, and ganglia appear, which consist primarily of unipolar neurons. The connection between two neurons is realized through a synapse. [2] A chain-like connection of the ganglia is formed, followed by their fusion in the head, and thus the segmental spinal cord and, in its anterior part, the brain are formed. Their rapid development was made possible by the fact that the neuron with multiple projections had become predominant in them. The spinal cord and brain fragments determine the development of the brain, and at the peak of evolution, due to the determinant nature of the cerebrum, humans appear.

Undoubtedly, the most significant stage of development is the appearance of the cerebral cortex. This is where the sensory information of the centers under the cortex flows in, it develops the appropriate motor responses, and learning processes are usually related to this as well. The cerebrum and especially the cortex grew particularly huge in humans. [3] Nine-tenths of the approximately 24 billion neurons in the human nervous system are in the cerebral cortex. It is estimated that an average of 9 trillion, extremely complex connections were developed between the neurons of the brain that have not yet been fully explored in detail. This, in principle, means the formation of a completely new nervous system structure, and it indicates that the cerebral cortex is not only the terminating area of sensory systems or the starting point of descending motor paths, but also the center of other higher neurological activities.

\section{BRAIN FUNCTIONS WITH UNLIMITED DIMENSION}

So how do the news from the outside and inside world get to the brain? In sensory cells (exteroreceptors, intero- and proprioceptors), stimuli create lesions that reach the center in the form of potential changes on neurons. The center of the nervous system, the brain - where the stimulus eventually flows in - is essentially nothing more than a very complex neural network. It is much more complex than the peripheral network, so it is no wonder that it has even more, completely new, features and related functions. [4]

Sensory cells and sensory organs have well-determined centers, quasi "representative sites" in the brain; the stimuli of a sensory organ always flow into the corresponding center. We also know that from there, they reach many other areas of the brain. 
Because the center receives a lot of stimuli at once, there are many neurons operating at all times. Depending on how many cells are stimulated in the brain at the same time and what brain cells are operating in space and time, ever-varying stimulus "patterns" are created. [5] It depends on these stimulus patterns - we do not yet know how - the subjective feeling of stimuli. And this feeling also means that the received, basically completely identical stimuli - of action voltage series - have already regenerated the original information, the feeling of the stimuli in the brain already imitates again, faithfully reflecting their different nature (color, smell, hardness, etc.).

The brain compares, groups, combines and stores information from the outside world and the internal world of the organism for a shorter or longer period of time. [6] These functions can be seen as sources of consciousness, thinking, behavior and memory. With the help of the information obtained in this way, it regulates and coordinates the functioning of the living organism in accordance with the incoming stimulus pulses.

\section{THE BRAIN AS QUANTITATIVE AND QUALITATIVE GROWTH}

Humans as systems are led by the brain in order to maintain a constant goal (it is about keeping themselves alive). So, it is a central organ that is responsible for reserving humans as systems and coordinating the various functions. Our brain, both awake and asleep, constantly controls and directs the biological functioning of our organisms.

The subsystems of our organism, in our opinion, are controlled by the "hypothetical secondary brains" and they only control and direct the only objective that characterizes the given subsystem. Thus, for example, in the case of the digestive apparatus, they only control the processes related to digestion. [7]

The human brain is a system $\left(\mathrm{S}_{\mathrm{i}}\right)$ that has an ontogenetic evolution, both structurally and functionally - from birth to death. In order for the brain to be examined from the perspective of systems theory, we need to introduce certain concepts, more precisely structural elements called topograms $\left(T_{j}\right)$ and functional elements called functiograms $\left(F_{k}\right)$. [8]

$\boldsymbol{i}$ is the time factor in the system, which is characterized by the set of natural numbers, at birth $\mathbf{i}=\mathbf{0}$. So, at birth, the human brain is an $\mathrm{S}_{0}$ system that contains $\mathrm{k}$ topograms and $\mathrm{h}$ functiograms.

Over time, two types of brain growth can be observed: quantitative and qualitative growth. Quantitative growth is made up of two parts: surface (which increases in proportion to the square of the distance) and volumetric (which increases with the cube of the distance). A significant part of the topograms are located on the surface, while the functiograms are located in space, therefore, in the case of quantitative growth, we usually find that the number of functiograms increases faster than that of the topograms. [9]

Quantitative growth alone is mainly characteristic of those parts of the brain that are called called the hypothetical secondary brain. These consist of constant (stable) topograms and functiograms born with us. During a person's life, they barely change, these provide the centers of the reflexes we are born with and the neural activities associated with them. Most are already found in the $\mathrm{S}_{0}$ state of the brain system. Temporary changes may occur in perturbed states, but usually negative feedbacks do their best to restore their normal homeostatic function. [10]
Qualitative growth of the brain means that temporary topograms and functiograms are created to operate conditioned reflexes. In humans, they also represent the centers of human communication related to the second signaling system (speech, writing, reading, learning, thinking, etc.). At the end of a person's life, this is where the degradation begins: a decrease in the number of neurons breaks down the structure of temporary topograms, a lack of repetition results in the loss of functiograms. So, as a result of these changes, the older a person is, the stronger their forgetfulness and the less effective their mental activity.

\section{LEARNING}

A single encoding, storing and retrieving is not yet sufficient to preserve the memory trace. To preserve it, we need to repeat this process several times, as the saying goes, "repetition is the mother of all learning". The problem of the frequency of repetitions over time arises here. The repetitions are distributed in time, and carried out over several occasions, because recalling what has been learned is always only possible on the basis of some kind of association.

How does the association take place in everyday life? When a child learns to speak, the tone of the spoken word is completely meaningless to them at first. However, if the word "table" is associated more than once, for example, with light or tactile or other stimuli conveying information about its shape, material, etc., after a certain number of repetitions the sound image of the word itself evokes the image of the table. We learn foreign languages through similar associations in our adulthood. [11]

Because stimulus patterns from the sensory organs run into different parts of the brain and settle in different areas, their connections can only be imagined as the stimulus spreads upon reception and settlement, and thus the different centers come into contact with each other. These relationships represent the physiological background and basis of the association, the process of memory. Since virtually any kind of stimuli can come into contact with each other, memory is based on the unified functioning of the entire brain. [12] Although there is no clear explanation of the biological mechanism of memory yet, we can gain psychological, medical, and pedagogical experiences from it that may be remarkably necessary and useful in our practical lives.

Learning and memory are a basic feature of the central nervous system. Our whole behavior is a learned process that evolved on the basis of unconditional reflexes and instincts, being built around them. Learning is closely related to other brain processes, especially attention and cortical activity. Based on these, the cortex is able to capture and process information in such a way that in the event of a repetition of the same stimulus, the response is consistent with the previous event. [13] In humans, learning is possible without an external event, through the mental recalling of events and the new logical association of concepts, given that memorization and the storage of information are mandatory conditions for any type of learning.

Learning is a phenomenon related to the cerebral cortex. The cerebral cortex is home to all the mechanisms capable of the fastest and most finely differentiated stimulus analysis, as well as the determination of the appropriate response. Similarly, the cerebral cortex is the site for comparing and storing data recorded on various information channels and stored in memory. Learning occurs only when the perceived information differs from what is stored, that is, as opposed to the individual's prior experience. 
Other areas of the central nervous system are also involved in the learning process: the limbic system, the thalamus, the reticular formation, all of which are necessary because they transmit sensing-sensory information to the cerebral cortex. During learning, a number of unstable function patterns are created and their existence over time is ensured by repetition.

Extremely complex learning mechanisms are the nervous and humoral ones. The basic neural mechanisms of learning are the following: the classical conditional reflex, respectively their formation (which is the simplest and most common type) and operant learning. The latter occurs when, intentionally, as a result of a certain activity, an individual is either rewarded or punished. Both mechanisms are based on differentiation processes and are modified through experience. They become important to learning only to the extent that change is made through experience. [14]

The morphological factor (substrate) of the learning process is less known. It is presumed that learning involves the development of synaptic connections, as repeated stimulation of individual neural structures results in an increase in the number of dendrites, swelling of the ends, and elongation and thickening of the axon terminals. The close relationship between neurons and glial cells, as well as the large number of glial cells, suggest the assumption that glial cells, when inserted between neurons, form synaptic bridges that facilitate the transmission of stimuli between two neurons. This assumption is supported by the finding that RNA content increases in stimulated neurons while it decreases in glial cells. Another hypothesis is that the nerve stimulus activates certain enzyme systems in the neurons it passes through, modifying the synthesis of certain peptides that would be material factors for learning and memory.

There are generally three types of learning strategies: indepth learning strategy: strives for understanding, grasping great connections, seeing over system; organized learning: regular learning, work organization; mechanical learning memorizing details, recalling as accurately as possible is the goal.

These strategies are mixed and are present in different proportions in the case of each student. It is important to note that all three strategies play a very important role in the learning ability, and neither method should be despised, however, the one we choose in a given situation and the one that predominates do make a difference. These three main learning methods are built on elementary learning techniques. Elementary learning techniques create complex learning strategies by forming a specific system.

\section{THINKING}

Thinking means the totality of intellectual activity (a typically human form of information processing); it belongs to cognitive functions along with perception, imagination and memory. In contrast to direct observation and an instinctive relationship with the environment, thinking means the ability to consciously represent the processes and realities of the internal and outside world. [15] Thinking as a process that requires meaning is the basis of human existence. We distinguish between concrete and abstract, subject-specific and conceptual, defining, judgmental and speculative, analytical (disintegrating) and synthetic, discursive (based on subjective abstraction) and intuitive, introspective, meditative, productive (creative) and reproductive thinking. All of these forms are encompassed by the concept of intelligence, along with other abilities. [16] Thinking means the need for assertiveness mediated by speech, for example, in terms of authenticity, correctness and credibility. Although thinking is closely tied to speech, in many cases it cannot be compared to it.
The brain compares, groups, combines and stores information from the outside world and the internal world of the organism for a shorter or longer period of time. These functions can be seen as sources of consciousness, thinking, behavior and memory. [17] With the help of the information obtained in this way, it regulates and coordinates the functioning of the living organism in accordance with the incoming stimulus pulses. The brain can also develop ideas, suggestions, solutions on the time scale of thought and action. In reality, it can also be interpreted as if our brains manipulate the information stored, create competing ideas, and the randomness of this competition results in the more effective or less effective guess. [18]

Humans carry out all these activities with their brain and through their so-called thinking activities. Thinking is the indirect form of cognition, in the course of which we gain new knowledge that has not yet been gained through direct experience.

\section{SUMMARY}

Humans as psychobiological systems are regulated by the nervous system. The development of the nervous system begins in the fetal age and it is during the inheritance that all its stable topograms and function diagrams that are the base of their involuntary reflexes solidify. Thus, at birth, we already have the cortical projections of the sensory and motor homunculus. The quantitative growth of the brain takes place in a similar way in the brain as well as in the so-called hypothetical brains. The significant difference occurs in qualitative growth, which is not characteristic of hypothetical secondary brains, while in the central nervous system it plays a decisive role. Qualitative growth creates the elements and conditions of psychic regulation in humans. It should be emphasized that there are differences in the qualitative growth of the two hemispheres of the brain that result in the dominance of certain skills throughout life. It is important to note that psychic regulation is superior to neural regulation.

\section{REFERENCES}

[1] Vincze, J. The Capital Chapter of Biophysics. $5^{\text {th }}$. Ed. NDP P., Budapest, 2015.

[2] Leake, M., Biophysics: Tools and Techniques. CRC P., Paris, 2016.

[3] Vincze, J.: Medical Biophysics. NDP P., Budapest, 2018.

[4] Cunningham,K. L., Littleton J. T.: Neurons regulate synaptic strength through homeostatic scaling of active zones. J. Cell Biol. 2019; 218(5) 1434-1445.

[5] Kumark, P.J., Clark, M.: Clinical Medicine. 10 ${ }^{\text {th }}$ Ed., 2019. Elsevier

[6] Vincze, J.: The Biophysics is a Borderland Science. Roumanian J. Applied Sciences Technology. 2020; 2(3):115-121.

[7] Vincze, J., Vincze-Tiszay, G.: The "hypothetical secondary brain", Medical Research Archives. 2019; 7(7):1-3

[8] Vincze, J., Vincze-Tiszay, G.: The Hypothetical Secondary Brain of the Digestive Apparatus.Int. J. Reas. Educ. Hum. Com., 2021; 4(1): 77-84.

[9] Vincze, J., Vincze-Tiszay, G.: The Hypothetical Secondary Brain of the Excretory Apparatus. Amer. J. Engin. Research, 2021; 10839. 32-37. 
[10] Vincze, J., Vincze-Tiszay, G.: The Hypothetical Secondary Brain of the Respiratory Apparatus. j. Med. Phamac. Sciences, 2021; 1(1):1-6.

[11] Matthews, W. J., Meck, W. H.: Temporal Cognition Connecting Subjective Time to Perception, Attention AND Memory. Psychol. Bull. 2016; 142(8): 865-907.

[12] Gerber, J.D., Whealer L., Suls, J.: A Social Comparison Theory Meta-Analysis. Psychol. Bull. 2018; 144(2): 177-197.

[13] Vincze, J.: The Biophysical Modeling of the Apparatuses in the Human Organism. Lambert Academic Publishing, Berlin, 2020.
[14] Vincze, J.: Biophysics of the Apparatuses in Human Organism. NDP. P., Budapest, 2020.

[15] Matthews, W. J., Meck, W. H.: Temporal Cognition: Connecting Subjective Time to Perception Attention, and memory. Psychological Bull. 2016; 142(8):865907.

[16] Vincze, J.: The Biophysics is a Borderland Science. NDP P., Budapest, 2015.

[17] Colling, R., Pitman, H., Dien, K. et al.: Artificial Intelligence in Digital Pathology. J. Pathology, 2019; 249(2):143-150.

[18] Vincze J.: Biophysical vademecum. NDP P. Budapest, 2021. 